\title{
TATA-binding Protein (TBP) Involved in the Ecdysone Signal Pathway of Drosophila
}

\author{
Gang Wang \\ Department of Genetics, Weifang Medical University, Weifang, 261053, China \\ Corresponding author, gangwt@126.com
}

Keywords: Drosophila; Ecdysone; TBP gene; Expression profile

\begin{abstract}
The Drosophila TATA-box binding protein (TBP) is a general transcription factor required by all three eukaryotic nuclear RNA polymerases, it can recognize the TATA-box sequence to initiate gene transcription. TBP and other TATA binding factors are spatio-temporal control of gene expression in different tissue types or in different stages of development. However, how the TBP gene is mediated is still elusive. This study reveals that TBP expression increased at molting and metamorphosis stage, 20-hydroxyecdysone (20E) can induce TBP expression, and the silence of EcR-A and USP1 led to a reduced transcript level of TBP in S2 cells. These results indicate that the TBP gene involved in the 20E signaling transduction pathway, and it acts downstream EcR and its heterodimer USP.
\end{abstract}

\section{Introduction}

The TATA-box binding protein (TBP) is a general transcription factor required by all three eukaryotic nuclear RNA polymerases, especially by RNA polymerase II [1]. The RNA polymerases can utilize TBP recognize and bind the TATA box, which is located about 30 bp upstream of transcription start of TATA-containing genes in mammalian [2]. The TBP is conserved from archaea to human; it has two major domains. The C-terminal domain is highly conserved, consists of two direct pseudo repeats and folds, and it can bind to the TATA box via an induced fit model. The TBP $\mathrm{N}$-terminal domain, which has a glutamine repeat region, is relatively variable in sequence and size between species [3, 4].

TBP is the founding member of TBP paralogs family in eukaryotic genomes, TBP and other TATA binding factors, such as TBP-related factor (TRF), TBP-like factor (TLF) and TBP2, are spatio-temporal control of gene expression in different tissue types or in different stages of development [5]. The TBP gene expressed various amounts at different development stages. For example, TBP mRNA is abundantly expressed in oocytes, but only translated into protein when early cleavage and the TBP protein levels get peak at the midblastula transiton in Xenopus [6]. Some researchs have indicated that the TBP expression levels also increased during infection or in transformed cells [3, 7]. However, the changes in TBP gene expression during the whole development process have not been studied in detail.

During insect development, ecdysone (20-hydroxyecdysone, 20E) play important role in regulating insect molting and metamorphosis. Upon binding of $20 \mathrm{E}$ to the ecdysone receptor (EcR) and the ultraspiracle (USP), a small set of early genes such as E74, E75 and broad complex (BR-C, encodes zinc finger proteins) were induced and help mediate molting and metamorphosis [8].

In order to investigate how TBP expressed during Drosophila development, and the relationship between 20E and TBP gene, we utilized quantitative real-time PCR, semi-quantitative reverse transcriptase PCR, and RNAi to analyze the Drosophila TBP expression profile. The results demonstrate that the TBP gene exhibiting higher expression level during molting and metamorphosis stages and it can be induced by the $20 \mathrm{E}$ signal transduction pathway through the EcR-A and its heterodimer USP1. 


\section{Materials and Methods}

Insect maintenance. The Drosophila were cultured in the laboratory with an artificial diet containing $5.2 \%(\mathrm{w} / \mathrm{w})$ glucose, $8.7 \%(\mathrm{w} / \mathrm{w})$ cornmeal, $0.3 \%$ antiseptic reagents, $3.5 \%(\mathrm{w} / \mathrm{w})$ dried yeast and $0.8 \%(\mathrm{w} / \mathrm{w})$ agar at $24 \pm 1{ }^{\circ} \mathrm{C}$ under $12: 12 \mathrm{~h}$ light and dark conditions.

Quantitative real-time PCR. The Drosophila larval body and S2 cells total RNA were isolated according manufacturer's instructions. Larvae were firstly homogenized with Unizol reagent. The RNA quality was detected and approximately $4 \mu \mathrm{g}$ total RNA was reverse transcribed into the first strand cDNA. Quantify the transcript levels by quantitative real-time PCR at different developmental stages and various treated Drosophila S2 cells. Based on the Drosophila TBP gene sequence (NM_079081.4), we designed the specific primers for quantitative real-time PCR, TBPF: 5'-cggcttaatctatcgtatgg-3', the reverse primer TBPR: 5'-atgactgcttcttgaacttc-3'. The act5C was used as internal standardization, the designed primers were: act5CF: 5'-ctggcttcgctgtccacctt- $3^{\prime}$ and act5CR 5'-gcactttcgctgctgcttcc-3'.

The quantitative real-time PCR performed in a volume of $10 \mu$ containing $2 \mu l$ forward and reverse primers $(1 \mu \mathrm{mol} / \mathrm{L}), 5 \mu 1$ of $2 \times \mathrm{SYBR}$ Premix EX TaqTM and $1 \mu \mathrm{l} \mathrm{cDNA}(1: 10$ diluted) as template. The PCR was programmed one cycle at $94{ }^{\circ} \mathrm{C}$ for $3 \mathrm{~min} ; 40$ cycles of $94^{\circ} \mathrm{C}$ for $15 \mathrm{~s}, 65^{\circ} \mathrm{C}$ for $25 \mathrm{~s}$. Using the comparative CT method to analyze the expression level of TBP gene, the discrepancy between the CT for the TBP gene and act5C $(\triangle \mathrm{CT})$ was calculated. The expression level of TBP gene was calculated by $2^{-{ }^{\mathrm{CT}}}$. Three independent experiments were performed and analyzed to construct a graph.

Semi-quantitative RT-PCR. The semi-quantitative RT-PCR was also performed for quantifying transcript levels after treated in Drosophila S2 cells. Firstly, we have monitored and ensure that the TBP, act5C, EcR-A and USP1 were analyzed at the amplification phase. The TBP and act5C use above specific primers. The primers for EcR-A was: EcRF: 5'-aacaacaattagctgcctct-3'; EcRR: 5'-gtcccttcttgctcttcttc-3'. For USP1, the primers were: USP1F: 5'-caacggttctgatgacttca-3' USP1R: 5'-gtaatgcggaagaggaacag-3'.

The RT-PCR was programmed one cycle at $94{ }^{\circ} \mathrm{C}$ for $3 \mathrm{~min} ; 28$ cycles of $94{ }^{\circ} \mathrm{C}$ for $30 \mathrm{~s}, 56{ }^{\circ} \mathrm{C}$ for $30 \mathrm{~s}$, and $72{ }^{\circ} \mathrm{C}$ for $25 \mathrm{~s}$, one cycle at $72{ }^{\circ} \mathrm{C}$ for $8 \mathrm{~min}$. The PCR products were detected on $1.5 \%$ agarose gels, analyzed by the Quantity One Software. The intensity ratios for EcR-A, USP1, TBP to act5C were analyzed according the above data. Three independent experiments were analyzed to construct a graph.

Hormonal regulation of TBP gene. 20-hydroxyecdysone ((20E, Sigma, St, Louis, MO) was dissolved in dimethyl sulphoxide (DMSO) at the storage concentration of $10 \mathrm{mg} / \mathrm{ml}$. The storage solution of $20 \mathrm{E}$ was diluted with phosphate buffered saline (PBS) prior to adding into S2 cells. The final concentration of hormone was $1 \mu \mathrm{M}$. The control cells using equal DMSO volume. Three independent experiments were analyzed to construct a graph.

RNAi in S2 cell line. Using the MEGAscript ${ }^{\mathrm{TM}}$ RNAi kit (Ambion, Austin, Texas), we synthesized the dsRNAs against EcR-A and USP1. All the PCR primers for synthesize dsRNAs has a T7 sequence (5'-gcgtaatacgact cactatagg-3') on the 5' end. The primers for synthesize EcR-A dsRNA was designed, EcRiF: 5'-T7-aacaacaattagctgcctct-3'; EcRiR: 5'-T7-gtcccttcttgctcttcttc-3'; for USP1, USP1iF: 5'-T7-caacg gttctgatgacttca-3' USP1iR: 5'-T7-gtaatgcggaagaggaacag-3'. We synthesized the green fluorescent protein (GFP) dsRNA as control, and the primer was GFPiF: 5'-T7-tggtcccaattctcg tggaac-3' GFPiR: 5'-T7-cttgaagttgacettgatgcc-3'. The quantity was detected by spectrophotometry at $260 \mathrm{~nm}$, and the dsRNA quality was detected by $1 \%$ agarose electrophoresis. The S2 cells were transfected according the Invitrogen instructions. The total RNA was isolated for RT-PCR analysis. Cells treated with dsGFP were set as control. Three independent experiments were analyzed to construct a graph. 


\section{Result}

Expression profile of TBP. To investigate how TBP expresses during the third instar larvae and pupal stage, the total RNA was extracted from the third instar larvae at $0 \mathrm{~h}$ to $60 \mathrm{~h}$ and pupae at $12 \mathrm{~h}$ to $72 \mathrm{~h}$. Quantitative real-time PCR analysis showed that the TBP mRNA level higher at $0 \mathrm{~h}$ of the third instar larval stage when larva undergoing molting, and decreased at $12 \mathrm{~h}$ until $48 \mathrm{~h}$ when the larvae undergoing feeding stage. During the pupal stage, the expression of TBP increased at $0 \mathrm{~h}$ until $48 \mathrm{~h}$, and then subsequently slight decrease at $60 \mathrm{~h}$ until $72 \mathrm{~h}$ (Fig. 1). We can conclude that TBP mRNA was abundantly expressed when molting and metamorphosis stage.

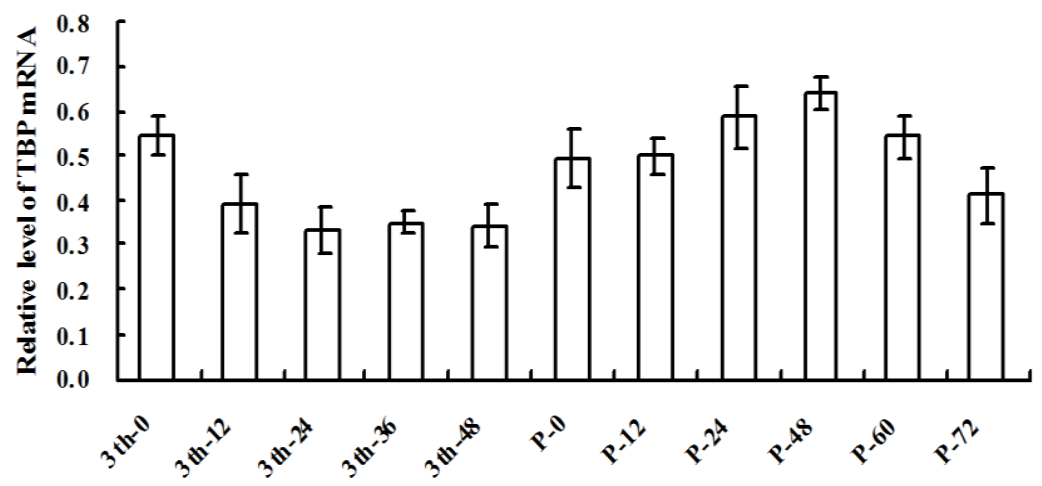

Figure. 1 Quantitative real-time PCR analysis of the TBP expression profile during the third instar larval and pupae development from the third instar larvae $0 \mathrm{~h}$ to pupae $72 \mathrm{~h}$; $a c t 5 c$ was set as the control. Error bars mean the standard deviation of three independent experiments.

Hormonal influences on TBP. To examine how 20E effect TBP expression, the S2 cells were cultured various times after the 20E was added. RT-PCR analysis results showed that the TBP mRNA level increased at $12 \mathrm{~h}$ after 20E treatment (Fig. 2). Thus, TBP gene can be induced by the ecdysone signal pathway.

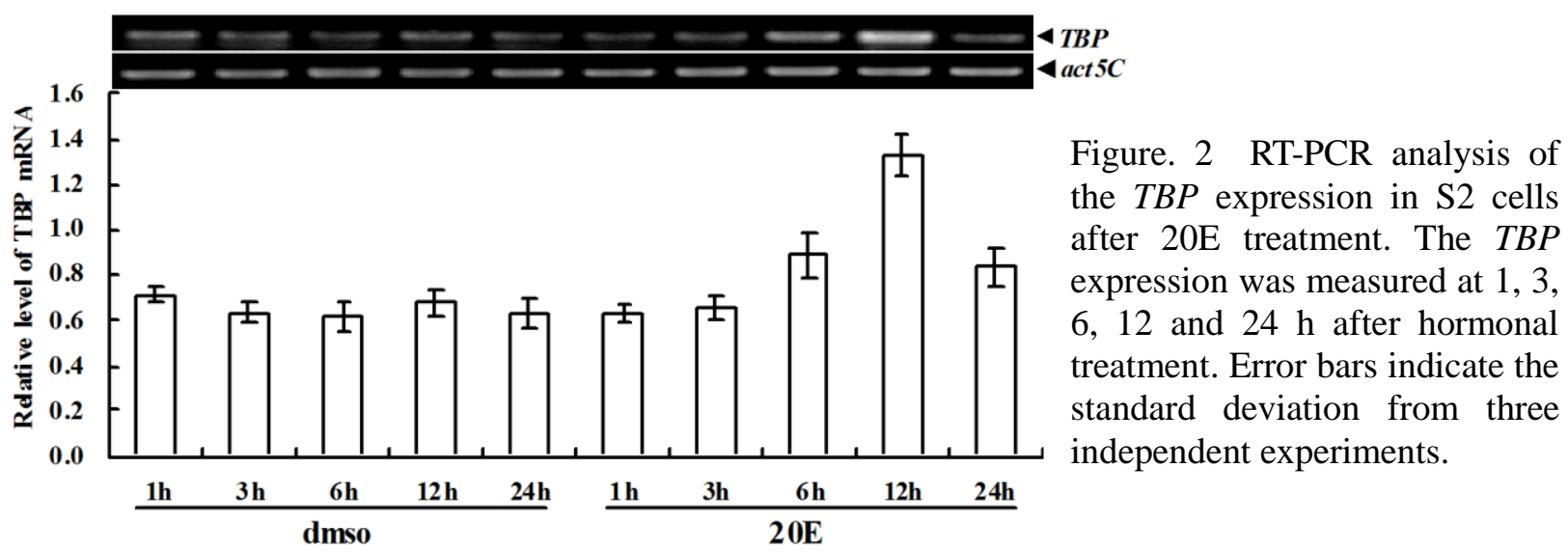

Silencing EcR-A and USP1 blocking the expression of TBP. To determine whether the USP and EcR are necessary for the induction of TBP by ecdysone, the USP1 and EcR-A were silenced by RNAi in S2 cells. Analysis showed that the silence of USP1 and EcR-A resulted in the suppression of TBP induction by $20 \mathrm{E}$ compared with the control which is transfected with dsGFP (Fig. 3). Those results suggest that TBP is involved in the ecdysone signaling transduction pathway and acts downstream of EcR and USP.
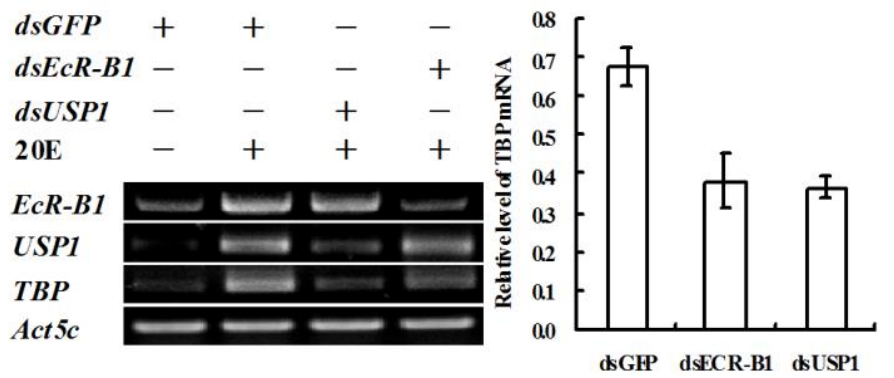

Figure. 3 RT-PCR analysis of the $T B P$ expression in $\mathrm{S} 2$ cells after $E c R-A$ and USPI silence. Error bars indicate the standard deviation from three independent experiments. 


\section{Discussion}

In this study, we investigated Drosophila TBP gene expression profile during the third instars larval and papal stages, the results showed that the TBP gene expressed with fluctuating variation, its expression increased when molting and metamorphosis. 20E can induce TBP expression in S2 cells, and the silence of USP1 and EcR-A led to a reduced transcript level of TBP gene. Those results indicated that TBP involve in the ecdysone signaling transduction pathway and acts downstream of EcR and USP.

During the holometabolous insects molting and metamorphosis, the integument is casted off, the larvae lose the old integument while the new one not becomes mature, then the insects dangerously exposed to attack by microorganisms. During this period, ecdysone upregulate some innate immunity genes protect insects is necessary $[9,10]$. While during infection, the TBP expression also increased [7], so we can speculate that ecdysone may upregulate innate immunity gene through the TBP.

TBP and other TATA binding factors, such as TRF, TLF and TBP2, are spatio-temporal control of gene expression in different tissue types or in different stages of development [5]. For example, TBP2 and TLF as initiation factor switching occurs on account of differential expression of these proteins in gametes of metazoans. During embryos, TBP, TBP2 and TLF initiate gene expression. In somatic cells, gene expression mainly dominated by TBP [3]. Our research showed that when larval molting and metamorphosis TBP expression also increased. During insect molting and metamorphosis ecdysone titer increase and through binding to the ecdysone receptor EcR and USP, a number of early transcription factors such as E74, E75 and BR-C are induced; subsequently, a lot of late genes are unregulated to help replace old integument with a new one during molting, and induce larval tissues undergo histolysis or organogenesis during metamorphosis [11]. The TBP may be the initiation factor induces the early transcription factors expression, but the detail mechanism still need further investigated.

During the holometabolous insects molting and metamorphosis, the integument is casted off, the larvae lose the old integument while the new one not becomes mature, then the insects dangerously exposed to attack by microorganisms. During this period, ecdysone upregulate some innate immunity genes protect insects is necessary $[9,10]$. While during infection, the TBP expression also increased [7], so we can speculate that ecdysone may upregulate innate immunity gene through the TBP.

\section{Acknowledgements}

This research was supported by the Scientific Funds for Outstanding Young Scientists of Shandong Province Award [BS2013SW030], the Natural Science Foundation of Shandong Province (ZR2012HL42) and the Project of the Shandong Province Higher Educational Science and Technology Program (J12LK01).

\section{References}

[1] Burley SK. The TATA box binding protein. Curr Opin Struct Biol 1996;6:69-75.

[2] Juven-Gershon T, Kadonaga JT. Regulation of gene expression via the core promoter and the basal transcriptional machinery. Dev Biol 2010;339:225-9.

[3] Akhtar W, Veenstra GJ. TBP-related factors: a paradigm of diversity in transcription initiation. Cell Biosci 2011;1:23.

[4] Zhao X, Herr W. A regulated two-step mechanism of TBP binding to DNA: a solvent-exposed surface of TBP inhibits TATA box recognition. Cell 2002;108:615-27.

[5] Muller F, Zaucker A, Tora L. Developmental regulation of transcription initiation: more than just changing the actors. Curr Opin Genet Dev 2010;20:533-40. 
[6] Gonlugur U, T EG. A Retrospective Analysis of Nutritional Parameters in Chronic Obstructive Pulmonary Disease between Sexes. J Clin Biochem Nutr 2007;41:175-8.

[7] Mainz D, Quadt I, Stranzenbach AK, Voss D, Guarino LA, Knebel-Morsdorf D. Expression and nuclear localization of the TATA-box-binding protein during baculovirus infection. J Gen Virol 2014;95:1396-407.

[8] Riddiford LM, Hiruma K, Zhou X, Nelson CA. Insights into the molecular basis of the hormonal control of molting and metamorphosis from Manduca sexta and Drosophila melanogaster. Insect Biochem Mol Biol 2003;33:1327-38.

[9] Candido-Silva JA, Zanarotti GM, Gallina AP, de Almeida JC. Developmental regulation of BhSGAMP-1, a gene encoding an antimicrobial peptide in the salivary glands of Bradysia hygida (Diptera, Sciaridae). Genesis 2007;45:630-8.

[10] Wang G, Liu PC, Wang JX, Zhao XF. A BTB domain-containing gene is upregulated by immune challenge. Arch Insect Biochem Physiol 2011;77:58-71.

[11] Stilwell GE, Nelson CA, Weller J, Cui H, Hiruma K, Truman JW, et al. E74 exhibits stage-specific hormonal regulation in the epidermis of the tobacco hornworm, manduca sexta. Dev Biol 2003;258:76-90. 